\title{
Qualitative and quantitative modifications of root mitochondria during senescence of above-ground parts of Arabidopis thaliana
}

\author{
Diego Darío Fanello*, Carlos Guillermo Bartoli, Juan José Guiamet \\ Instituto de Fisiología Vegetal (INFIVE), Facultades de Ciencias Agrarias y Forestales y de Ciencias Naturales y Museo, Universidad Nacional de La Plata, \\ CCT-CONICET La Plata, cc 327, 1900, La Plata, Argentina
}

\section{A R T I C L E I N F O}

\section{Article history:}

Received 24 August 2016

Received in revised form 18 January 2017

Accepted 21 January 2017

Available online 7 February 2017

\section{Keywords:}

Mitochondria

Root

Senescence

Carbohydrate starvation

Respiration

Arabidopsis thaliana

\begin{abstract}
A B S T R A C T
This work studied modifications experienced by root mitochondria during whole plant senescence or under light deprivation, using Arabidopsis thaliana plants with YFP tagged to mitochondria. During postbolting development, root respiratory activity started to decline after aboveground organs (i.e., rosette leaves) had senesced. This suggests that carbohydrate starvation may induce root senescence. Similarly, darkening the whole plant induced a decrease in respiration of roots. This was partially due to a decrease in the number of total mitochondria (YFP-labelled mitochondria) and most probably to a decrease in the quantity of mitochondria with a developed inner membrane potential ( $\Delta \Psi \mathrm{m}$, i.e., Mitotracker redlabelled mitochondria). Also, the lower amount of mitochondria with $\Delta \Psi \mathrm{m}$ compared to YFP-labelled mitochondria at $10 \mathrm{~d}$ of whole darkened plant, suggests the presence of mitochondria in a "standby state". The experiments also suggest that small mitochondria made the main contribution to the respiratory activity that was lost during root senescence. Sugar supplementation partially restored the respiration of mitochondria after $10 \mathrm{~d}$ of whole plant dark treatment. These results suggest that root senescence is triggered by carbohydrate starvation, with loss of $\Delta \Psi \mathrm{m}$ mitochondria and changes in mitochondrial size distribution.
\end{abstract}

(C) 2017 Elsevier B.V. All rights reserved.

\section{Introduction}

The term senescence refers to the functional and structural deterioration observed in plants during the final stages of their development [1]. Monocarpic senescence involves different physiological processes occurring after flowering, including the remobilization of mineral and organic compounds from senescing leaves to growing fruit and seeds [1]. Senescence has been exhaustively studied in leaves and fruits but scarcely in other organs such as roots. Since much of the focus was on leaf senescence, most studies concentrated on chloroplasts since these organelles are the main source of carbon and nitrogen remobilized from leaves to other organs $[2,3]$. Knowledge about changes in other organelles, and particularly in mitochondria, is still very limited.

Mitochondria participate in primary metabolism involving carbon- and nitrogen-containing compounds. The synthesis and exchange of ATP/ADP, redox compounds and carbon skeletons constitute the main functions carried out by plant mitochondria [4-6]. These functions are considered critical for the proper dis-

\footnotetext{
* Corresponding author.

E-mail address: ddfanello@gmail.com (D.D. Fanello).
}

mantling of chloroplasts and translocation of nutrients from the leaves to other organs during senescence [7,8]. The number of mitochondria (calculated on the basis of cell volume) decreases during leaf senescence, in either photosynthetic (i.e., mesophyll) or non- photosynthetic leaf cells (i.e., epidermal cells) [8]. The respiratory activity of senescent leaves decreases as a consequence of a reduction in the amount of mitochondria when the whole plant is subjected to dark conditions [8]. However, when only one leaf is darkened and the rest of the plant is kept under light, the specific activity ( $\mathrm{nmol} \mathrm{O}_{2} \mathrm{~min}^{-1} \mathrm{mg}^{-1}$ protein) of remaining mitochondria stays constant [8].

Light deprivation abolishes photosynthesis, leading to a decrease of carbohydrate levels, which affects mitochondrial metabolism. The impact of decreased carbohydrate levels on mitochondria was studied using different experimental models, such as roots detached from the plant [9], plant cells in culture [10,11] and in vitro culture of apical root sections [12].

Some evidences suggest an association between mitochondrial morphology and carbohydrate starvation; spherical mitochondria were observed in cultured cells under normal conditions, whereas "enlarged" mitochondria were predominant under starvation [11]. Zottini and cowokers showed morphological changes in mitochondria of Medicago truncatula during different phases of protoplast 
culture [13]. These cells had giant mitochondria during the initial stages of senescence. In addition, different mitochondrial fractions obtained from sections of maize root apexes showed particular metabolic and structural properties and a reduced amount of mitochondria with low density when roots were subjected to carbohydrate starvation [14].

Alterations in mitochondria were observed during programmed cell death (PCD) and might be important for the progress of this process [15]. However, there are few analysis focusing on the behavior of plant mitochondria during senescence, and much less in roots. Several works have studied the relationship between changes in morpho-functional aspects of mitochondria and PCD [16-21]. However, senescence of organs, such as leaves, appears to differ from PCD in a number of significant ways. Cells in senescing organs undergo a gradual, orderly disassembly, the cytoplasm does not "bleb", and senescence can be reversed in various ways, contrary to PCD [22].

The main objectives of this work were to study quantitative and qualitative changes in root mitochondria during developmental senescence of above-ground organs of plants (mainly the leaves) and senescence induced by darkness, and to relate these changes to carbohydrate deprivation.

\section{Material and methods}

\subsection{Plant material and growth conditions}

Arabidopsis thaliana (Heyn.) ecotype Col-0, transformed with the yellow fluorescent protein (YFP) targeted to mitochondria under control of the 35S promoter (mt-YFP lines, [23]) was used in all experiments. Plants were grown in a hydroponic system as described by Norén et al., with modifications [24]. The nutrient solution consisted of $5.0 \mathrm{mM} \mathrm{Ca}\left(\mathrm{NO}_{3}\right)_{2}, 5.0 \mathrm{mM} \mathrm{KNO}_{3}, 2.0 \mathrm{mM}$

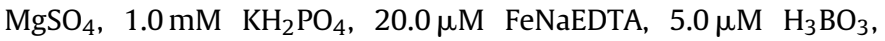
$0.9 \mu \mathrm{M} \mathrm{MnCl}_{2}, 0.8 \mu \mathrm{M} \mathrm{ZnCl}_{2}, 0.3 \mu \mathrm{MCuSO}_{4}$ and $0.01 \mu \mathrm{M} \mathrm{Na}_{2} \mathrm{MoO}_{4}$, pH 5.5-6.5 as described by Leggett and Frere [25]. Seeds were sown in $200 \mu \mathrm{L}$ pipette tips sealed at the base and filled with $50 \%$ nutrient solution in $0.7 \%(\mathrm{w} / \mathrm{v})$ agar. Tips were arranged in a closed rack containing a wet paper towel to keep humidity high. The rack was placed at $4{ }^{\circ} \mathrm{C}$ in darkness for 4 days. Then it was transferred to a growth chamber, with a short-day photoperiod ( $10 \mathrm{~h}$ light $/ 14 \mathrm{~h}$ dark) for darkness assays and long days ( $16 \mathrm{~h}$ light/8 h dark) for natural senescence assays. Irradiance was $120 \mu \mathrm{mol} \mathrm{m}{ }^{-2} \mathrm{~s}^{-1}$ (PPFD) and day/night temperature was $22 / 17^{\circ} \mathrm{C}$. Seeds were germinated and kept under these conditions for 10 days. Then pipette tips were cut off at the base and placed with their bases submerged in nutrient solution in a plastic container sealed with plastic film for 2 weeks. Finally, tips were placed in individual pots containing $400 \mathrm{~mL}$ of nutrient solution with air bubbling. Plants were grown for 4 weeks before starting the darkness incubation assays. Nutrient solution was replaced every 2 weeks (as the plants grew larger, more than 15-20 leaves, nutrient solution was replaced more frequently).

\subsection{Induction of senescence and glucose treatment}

For whole plant natural senescence experiments, the stages of Arabidopsis development were established according to rosette and inflorescence growth progression. In other experiments, senescence was induced by transferring Arabidopsis plants to continuous darkness for 5 or $10 \mathrm{~d}$, with control plants maintained under normal light conditions. Glucose treatment was performed by incubating plants in nutrient solution with the addition of 0.1 M glucose (+glu), with control plants without glucose (-glu) added. An antibioticantimycotic mixture consisting of $50 \mathrm{mg} / \mathrm{l}$ ampicillin [26] and
$250 \mu \mathrm{g} / \mathrm{l}$ amphotericin [9] was applied to prevent microbe proliferation.

\subsection{Measurement of root respiration}

Whole plant root respiration was measured by placing the root system in an air-tight chamber equipped with a Clark type $\mathrm{O}_{2}$ electrode at $25^{\circ} \mathrm{C}$ (Hansatech, UK). Prior to the measurements, roots were gently washed with deionized water and then blotted with paper towels to remove excess water. Root respiration, as well as other parameters (e.g., sugar content) was expressed on the basis of fresh weight because percentage of dry matter did not change during root ontogeny (data not shown). For measurements of respiration, the entire rosette or samples of the inflorescence (70-170 mg fresh weight for 4 week-old plants, 200-400 mg thereafter) were placed in an air-tight chamber.

\subsection{SDS-PAGE and inmunobloting}

Roots were harvested, frozen in liquid $\mathrm{N}_{2}$ and kept at $-80^{\circ} \mathrm{C}$ until analysis. Extracts ( $200 \mathrm{mg}$ root fresh weight/mL buffer) were made in $0.1 \mathrm{M}$ bicine buffer, pH 7.5, $1 \mathrm{mM}$ EDTA, 10\% v/v glycerol, $4 \mathrm{mM}$ cysteine, $1 \% \mathrm{w} / \mathrm{v}$ PVPP and protease inhibitors $(0.004 \%$ $\mathrm{w} / \mathrm{v}$ PMSF and $0.001 \% \mathrm{w} / \mathrm{v}$ Leupeptin). Homogenates were centrifuged at $1500 \mathrm{~g}$ and $4{ }^{\circ} \mathrm{C}$ for $20 \mathrm{~min}$ and the supernatants were collected for subsequent analysis. Soluble proteins were analyzed according to the protocol described by Laemmli [27]. Supernatants were mixed $(1 / 1 \mathrm{v} / \mathrm{v})$ with sample buffer $2 \mathrm{x}$ consisting of $125 \mathrm{mM}$ Tris- $\mathrm{HCl} \mathrm{pH} 6.8,10 \%(\mathrm{v} / \mathrm{v})$ glycerol, 4\% (w/v) SDS, 10\% (v/v) 2- $\beta$ mercaptoethanol, $6 \mathrm{M}$ urea, and $0.005 \%(\mathrm{w} / \mathrm{v})$ bromophenol blue. Samples were loaded on a $2 \mathrm{~mm}$ thick, $12 \%(\mathrm{w} / \mathrm{v}$ ) denaturing polyacrylamide gel and electrophoresed at $20 \mathrm{mAgel}^{-1}$ for $2.5 \mathrm{~h}$. For western blotting, proteins were electro-transferred to a nitrocellulose membrane at $200 \mathrm{~mA}$ for $1 \mathrm{~h}$. Blots were blocked in $5 \%$ $(\mathrm{w} / \mathrm{v})$ non-fat dry milk dissolved in PBS $(10 \mathrm{mM}$ phosphate buffer, $\mathrm{pH} 7.4,2.7 \mathrm{mM} \mathrm{KCl}$, and $137 \mathrm{mM} \mathrm{NaCl}$ ) and probed with specific antibodies for mitochondrial proteins: Cytochrome $c$ oxidase (cox2; [28]), cytochrome c (cytC; catalogue no. 554002, BD Pharmigen, USA) and L-Galactone-1,4-lactone dehydrogenase (L-GalLDH; [29]). After washing the membrane three times for $10 \mathrm{~min}$ each with PBS plus Tween 20 0.05\% (w/v, PBS-T), blots were incubated with a secondary antibody (goat anti-rabbit or anti-mouse IgG conjugated to horseradish peroxidase, for polyclonal or monoclonal antibodies, respectively) for $1 \mathrm{~h}$ and washed with PBS-T. Blots were developed by incubation in $10 \mathrm{~mL}$ of chemiluminescence mixture $(0.23 \mathrm{mM}$ coumaric acid, $1.2 \mathrm{mM}$ luminol dissolved in DMSO and

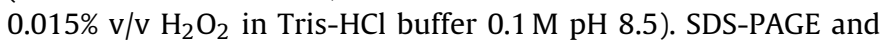
Westerns blots were analyzed with the ImageJ software, Analyze Gel function (1.46r version). For protein quantitation, the highest value in each image (Gel or Western blot) was used as a reference (and set as 1 ), and then all other bands in the same gel were referred to this. In all cases the reference $(=1)$ was the control replicate with the highest protein content, with other control replicates having smaller values. For this reason, the average values for controls are lower than 1.

\subsection{Confocal laser microscopy and staining procedures}

Fresh root samples were observed using an SP5 inverted confocal fluorescence microscope (Leica, Mannheim, Germany) with a $60 \mathrm{x}$ oil immersion objective. Mitochondria were detected by YFP fluorescence, and mitochondria with an inner membrane potential $(\Delta \Psi \mathrm{m})[30,31]$ were observed with Mitotracker Red CMXRos (MT, Molecular probes, USA). This dye was applied by incubating root sections in a $0.5 \mu \mathrm{M}$ MT solution for $20 \mathrm{~min}$; then, root sections were washed 3 times with deionized water and finally 
observed with the confocal laser microscope. Excitation/emission settings were $514 / 524-550 \mathrm{~nm}$ for YFP and $543 / 596-680 \mathrm{~nm}$ for MT. Quantitation of mitochondria density (i.e., number of discrete areas showing YFP and/or MT signal per root section observed under the microscope) was carried out with the Image software (version 1.46r). The procedure included: selection and measurement of root area, determination of threshold level, quantification of number and size of discrete areas with YFP or MT signal (with the particle analysis feature) and analyses of data in a spreadsheet. Colocalization analysis was performed using Manders coefficient (M) where: M1 (YFP $\rightarrow$ MT) corresponds to the proportion of YFP signal overlapping with MT and M2 (MT $\rightarrow$ YFP) corresponds to the proportion of MT signal overlapping with YFP. The JACoP Plugin (Just Another Co-localization Plugin, [32]) was used for co-localization analysis.

\subsection{Soluble sugar and starch measurements}

Soluble sugar measurements were made with the Anthrone method [33] using an ethanol extraction from fresh root tissues according to the protocol described by Costa et al. [34]. Starch measurements were performed in the pellet after ethanol extraction according to the protocol described by Rose et al. [35] (enzymatic method 2).

\subsection{Statistical analysis}

Data were analyzed using the Student's $T$-test and ANOVA tests and results were considered as statistically different when $P \leq 0.05$ $\left({ }^{*}\right)$. When variance (Levene test $P \geq 0.05$ ) or normality (Skewness Normality of Residuals, Kurtosis Normality of Residuals or Omnibus Normality of Residuals test $P \geq 0.05$ ) were regarded as different, a Kruskal-Wallis test was applied.

\section{Results}

\subsection{Root respiration during plant development}

Initial experiments were carried out to study root respiration and its changes during normal post-bolting development and senescence under a photoperiod of $16 \mathrm{~h}$. Bolting started 4 weeks after planting. Plants lost their rosette leaves (i.e., they completely dried out) after 13 weeks post planting, while inflorescence shoots remained alive, and roots retained their biomass even 21 weeks post planting (Fig. 1a). Roots maintained their respiratory activity $\left(\mu \mathrm{mol} \mathrm{O}_{2} \mathrm{~g}^{-1} \mathrm{FW} \mathrm{h}^{-1}\right.$ ) until late stages of plant development, with a significant drop only at 26 weeks (Fig. $1 \mathrm{~b}$ ).

\subsection{Effects of darkness on rosette and root biomass}

The decrease in root respiration following the death of leaves (rosette and cauline) might be a consequence of photoassimilate

a)

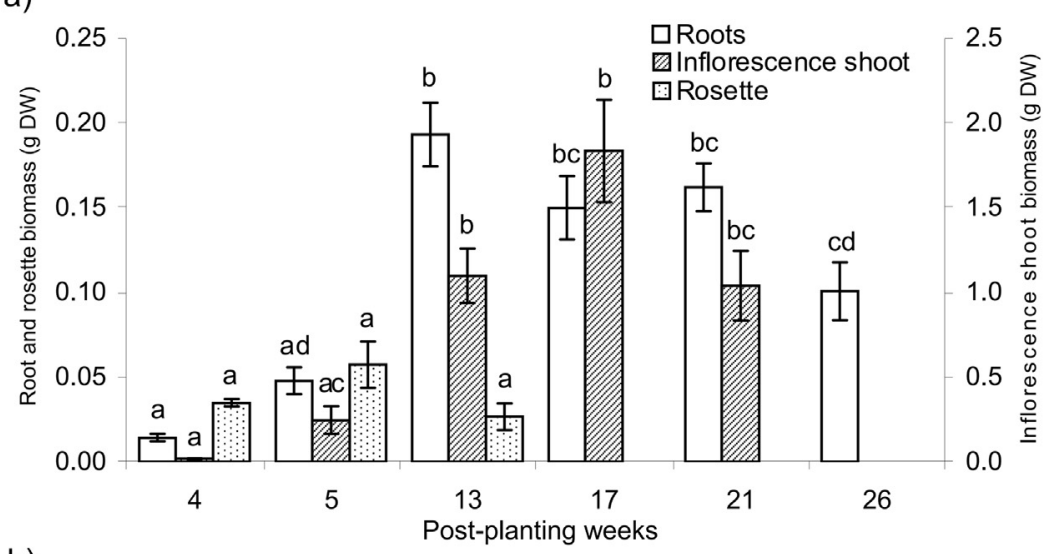

b)

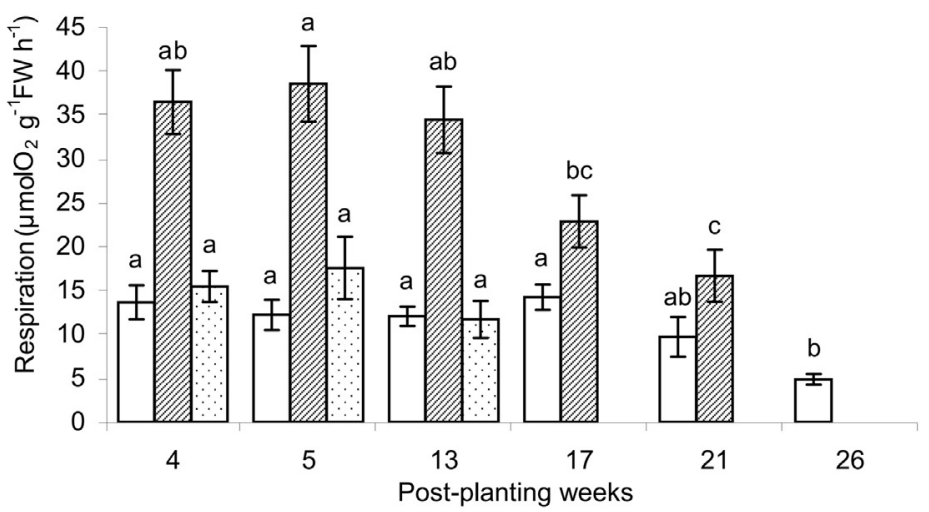

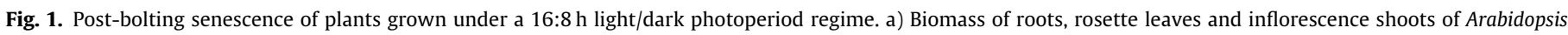

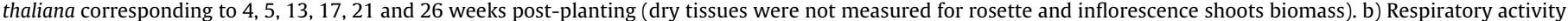

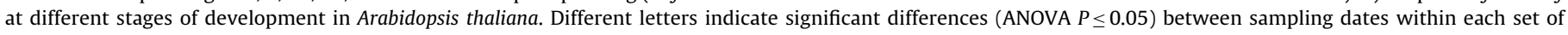

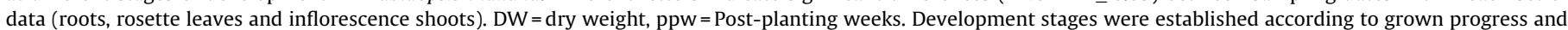

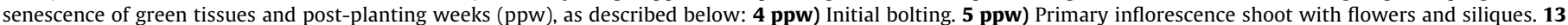

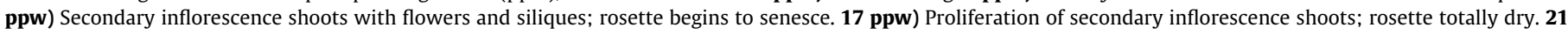
ppw) Much of the inflorescence shoots dry. 26 ppw) Shoots almost completely dry. Suplementary Fig. S1 provides images of plants. 


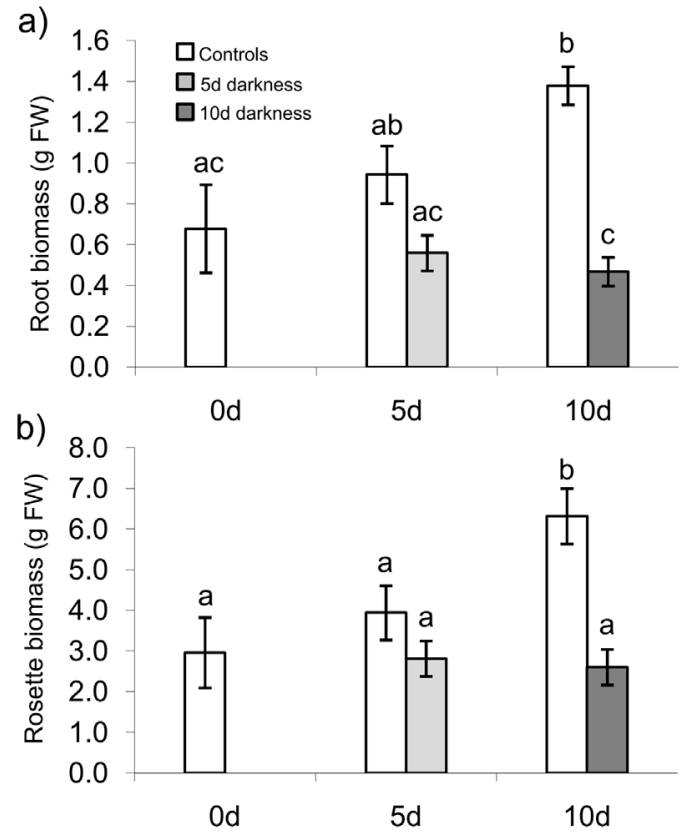

Fig. 2. Effects of light deprivation on the shoot and root biomass of plants subjected to 5 or 10 days of darkness ( $5 \mathrm{~d}$ or $10 \mathrm{~d}$, respectively) and control plants kept under a 10:14 h light/dark photoperiod regime. a) Root and b) Rosette. Empty bars represent controls kept at light/dark regime, light gray bars represent $5 \mathrm{~d}$ of darkness and dark gray bars represent $10 \mathrm{~d}$ of darkness. Different letters indicate significant differences (ANOVA $\mathrm{P} \leq 0.05$ ). $\mathrm{FW}=$ fresh weight. Suplementary Fig. S2 provides images of plants.

deprivation. To test this hypothesis, and to mimic possible carbohydrate starvation conditions during the post-bolting development of plants, root respiration was measured in plants incubated in protracted darkness. Both roots and rosette leaves ceased to grow on day 5 after the initiation of the dark treatment (Fig. 2a and b, respectively).

\subsection{Respiratory activity and protein content in roots of dark treated plants}

Root respiration decreased after $5 \mathrm{~d}$ of dark treatment compared with plants under normal light conditions (Fig. 3). It is important to mention that plants maintained under light displayed a constant respiratory activity during the 10d-period the experiments lasted indicating that differences observed were the consequence of dark treatment and not due to growing conditions or plant ontogenetic changes.

Similar trends were observed for total and mitochondrial protein contents (Fig. 4). The differences between $5 \mathrm{~d}$ and $10 \mathrm{~d}$ in both total and mitochondrial protein content of roots from dark treated plants were not significant (Student's $T$-test $P \geq 0.05$ ) except for LGalLDH.

\subsection{Quantification of mitochondria by confocal microscopy}

Typical images of YFP and MT-labelled root mitochondria (i.e., areas showing YFP and/or MT signal) are shown in Fig. 5a. A decrease in the abundance of mitochondria marked with YFP was observed in tangential optical planes of cortical root cells after $10 \mathrm{~d}$ of darkness treatment (Fig. 5b). Similarly, the amount of mitochondria detected with MT (mitochondria with a $\Delta \Psi \mathrm{m}$ ) showed a pronounced decrease after $10 \mathrm{~d}$ in darkness, compared to $5 \mathrm{~d}$ in darkness and light controls (Fig. 5b). The co-localization analysis shows that most of the mitochondria remaining after $10 \mathrm{~d}$ in darkness do not maintain a $\Delta \Psi \mathrm{m}$ (i.e., M1 coefficient indicates that most of the YFP signal does not co-localize with MT signal at 10d) (Fig. 5c).

An analysis of the distribution of root mitochondria classified according to their section size (i.e., the size of discrete areas displaying YFP and/or MT signals) using intervals of $1 \mu \mathrm{m}^{2}$ (Fig. 6) showed a decrease of YFP-labelled mitochondria abundance in the range of $0.5-1.5$ and $1.5-2.5 \mu \mathrm{m}^{2}$ sizes after $10 \mathrm{~d}$ of darkness (Fig. 6a). A large decrease of MT-marked mitochondria, especially in the range of $0.5-1.5$ and $1.5-2.5 \mu \mathrm{m}^{2}$, was also detected in the same roots (Fig. 6b).

\subsection{Starch and soluble sugar content and the effects of glucose supplementation}

To determine the effect of dark treatment on carbohydrates, the levels of starch and soluble sugars in the roots were measured. Starch content was not significantly affected by the dark treatment, either at 5 or $10 \mathrm{~d}$ (Fig. 7a). However, the content of soluble sugars decreased by about $50 \%$ in darkened plants after $5 \mathrm{~d}$ of treatment (Fig. 7b). The addition of $0.1 \mathrm{M}$ glucose for $24 \mathrm{~h}$ produced an increase in the respiratory activity of roots of all treatments (Fig. 8). No changes were observed in the abundance of either YFP

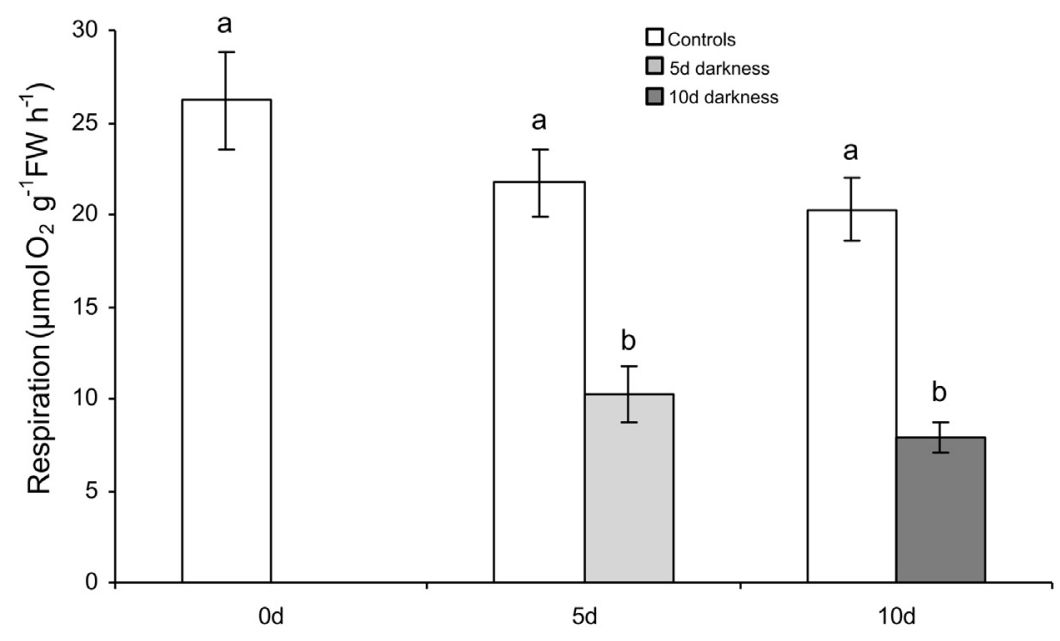

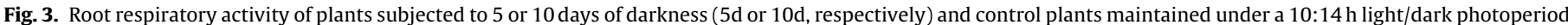

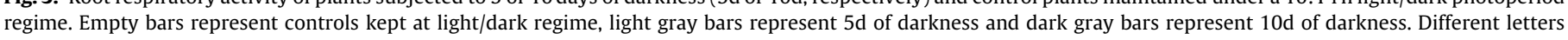
indicate significant differences (ANOVA $P \leq 0.05$ ). 


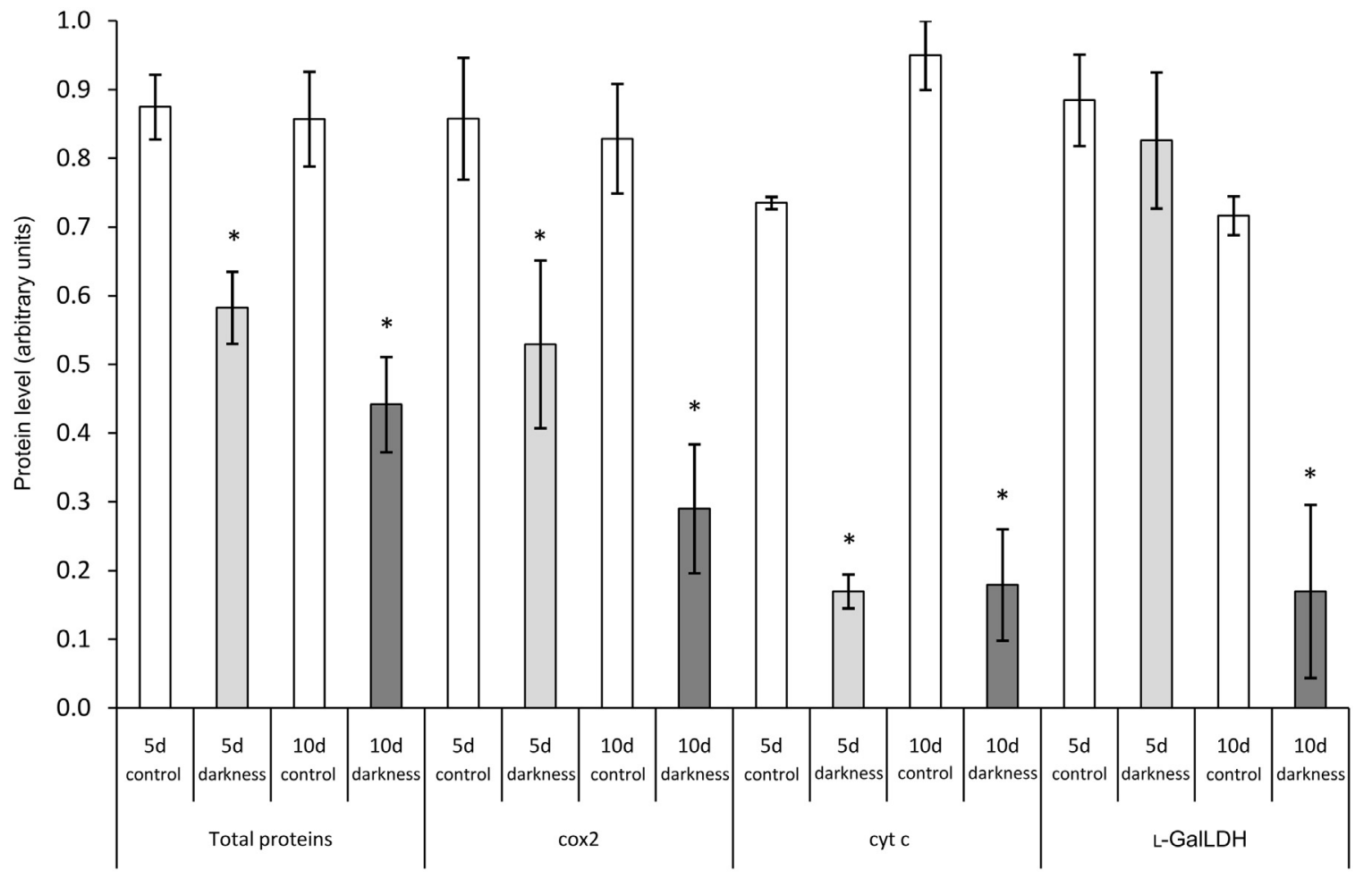

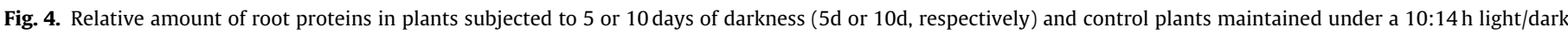

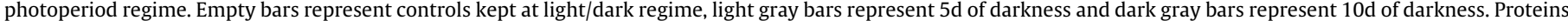

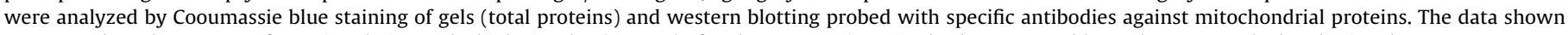

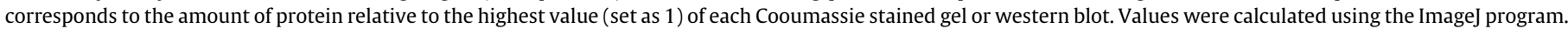

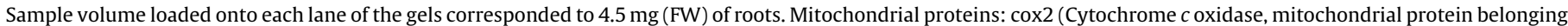

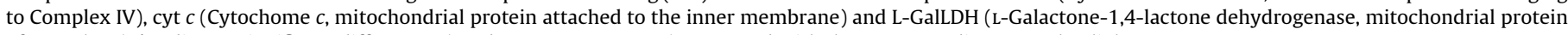
of Complex I). * Indicates significant differences (Student's $T$-test $P \leq 0.05$ ) compared with the corresponding control at light.

or MT-labelled mitochondria between +glu and -glu treatments (Supplementary Fig. S3).

\section{Discussion}

\subsection{Modifications in the quantity and activity of root} mitochondria under continuous darkness

A drop in respiratory activity was observed in roots during the progress of post-bolting senescence (Fig. 1). Changes in either the quantity of mitochondria and/or the $\Delta \Psi \mathrm{m}$ of mitochondria might account for changes in respiration. Both the decrease in the amount of YFP-labelled mitochondria and the even more pronounced decrease in the amount of mitochondria with $\Delta \Psi \mathrm{m}$ (i.e., labelled by MT) are consistent with the decrease in the contents of mitochondrial proteins and $\mathrm{O}_{2}$ uptake. However, the abundance of mitochondria with $\Delta \Psi \mathrm{m}$ decreased at $10 \mathrm{~d}$ of darkness, while $\mathrm{O}_{2}$ uptake did not change between 5 and 10 days in darkness. It is important to note that Mitotracker Red probes $\Delta \Psi \mathrm{m}$ but not all mitochondrial activities, and consequently, other mitochondrial functions might persist after 10 days in darkness. It was shown that mitochondrial integrity is conserved until the latest stages of leaf senescence, while their number drops by $30 \%$ [36]. On the other hand, some proteins that contribute to the modulation of the membrane potential in response to stress could explain the decrease of $\Delta \Psi \mathrm{m}$ in roots of senescent plants. MLS1 (MscS-Like, a family of mechanosensitive ion channels) leads to higher $\Delta \Psi \mathrm{M}$ in the presence of respiratory substrates but no ADP, and it may provide a mechanism for maintenance of mitochondrial redox status under stress [37]. Also, UPCs (uncopling proteins) linked to the dissipation of the proton gradient were found down-regulated in senescing leaves [36].

Although the amount of mitochondria may be indicated by units of volume, the quantification by unit of cross sectional area is a viable approach since mitochondria are discrete organelles [38]. The amount of mitochondria and $\mathrm{O}_{2}$ consumption do not always show a positive correlation. Under conditions of high $\mathrm{CO}_{2}$ concentrations, the amount of mitochondria increases but their respiratory activities decrease in several plant species [39]. These results indicate that changes in $\mathrm{O}_{2}$ consumption could not be attributed solely to the number of mitochondria (Figs. 9-11).

Since the fluorescence emitted by the MT probe depends on the mitochondrial membrane potential [30], the differences in the abundance of mitochondria labelled by YFP and MT might be related to a decrease in their metabolism, where most $\mathrm{O}_{2}$ uptake would be linked to uncoupled respiration. In soybean seedling roots, the amount of AOX increases with age but alternative path activity (oxygen consumption in the presence of $\mathrm{KCN}$ ) declined with root age [40] and it has been observed that AOX is synthesized de novo during aging of potato tubers [41]. Also, in tobacco cell cultures the induction of AOX could prevent events triggering cell death [42]. It is possible that some alternative path activity would play a role in maintaining root basal respiration in prolonged darkness.

For leaves, Keech et al. [8] found similar results to those shown here for roots, with a decrease in the amount of mitochondria and their metabolic activity becoming evident in leaves after $6 \mathrm{~d}$ of the initiation of dark incubation. In clover roots detached from the plant, $\mathrm{O}_{2}$ consumption did not change between the first 7-12 days but the quantity of total protein decreased [9], which is compara- 
a)

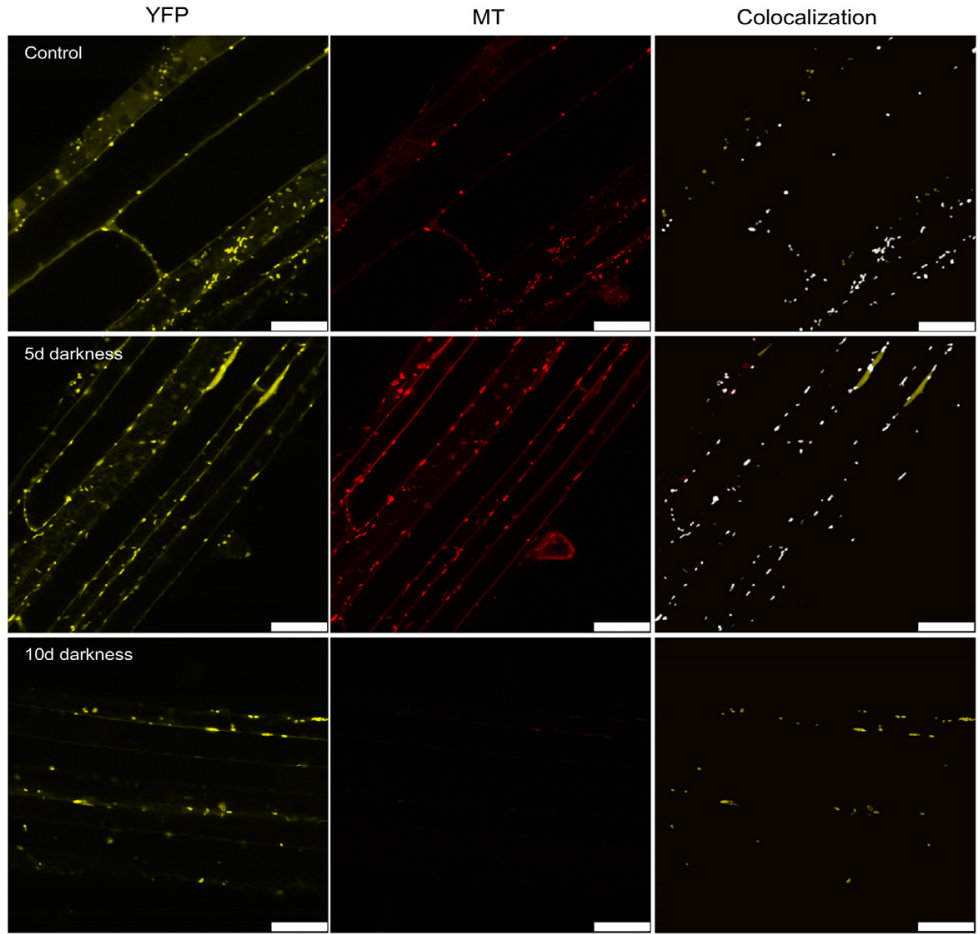

b)

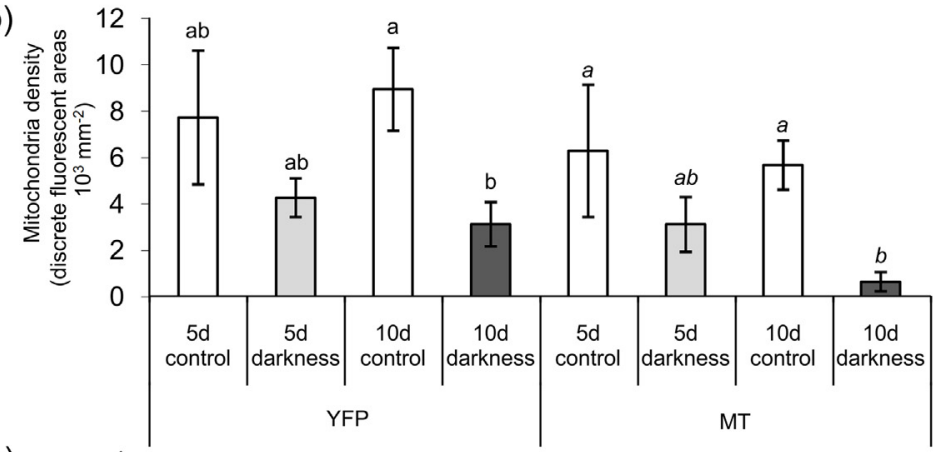

c)

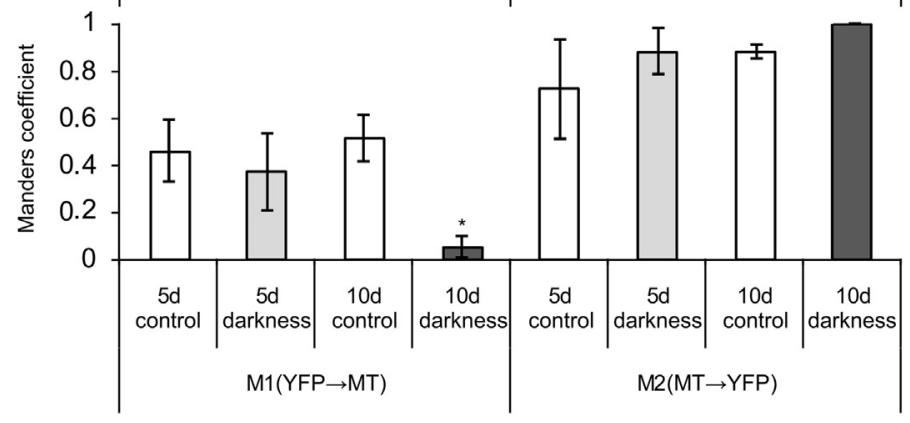

Fig. 5. Mitochondrial abundance in root cells calculated from the analysis of confocal images of plants subjected to 5 or 10 days of darkness (5d or $10 \mathrm{~d}$, respectively) and control plants maintained under a 10:14 h light/dark photoperiod regime. a) Confocal microscopy images. Since there were no differences for control roots between 0,5 and 10d, only the 0d control is shown. Colocalization images show overlapped signals between YFP and MT (Mitotracker red) channels in white. Images show cortex cells in a tangential view. Scale bars represent $25 \mu \mathrm{m}$. b) Number of mitochondria corresponding to YFP signal and MT (Mitotacker red) signal. Different letters indicate significant differences $(P \leq 0.05)$ according to the Kruskal-Wallis test. Normal font letters correspond to YFP differences and italics correspond to MT differences. $c)$ Results of the colocalization analysis as estimated through Manders coefficient. This coefficient varies between 0 (no overlap) and 1 (full overlap) where M1 (YFP $\rightarrow$ MT) corresponds to the overlap of the YFP signal on the MT signal, and M2 (MT $\rightarrow$ YFP) corresponds to the overlap of MT on the YFP signal. *Indicates significant differences (Student's T-test $P \leq 0.05$ ) between plants subjected to 5 or 10 days of darkness and control plants.

ble to the results observed here during the 5 th to the 10 th $\mathrm{d}$ of dark treatment applied to the whole plant.

In maize root tips there are mitochondrial fractions of different buoyant density with particular metabolic and structural properties, such as $\mathrm{O}_{2}$ uptake, protein content, enzyme activity and quantity of internal membrane cristae [14]. These mitochon- drial fractions differ between glucose-starved and non-starved root tips [14]. Mitochondrial pools with or without $\Delta \Psi \mathrm{m}$ (YFP vs MTlabelled mitochondria) or even, mitochondria with different sizes (i.e. different cross sectional area) shown in this work, could be related with these different mitochondrial fractions. However, this hypothesis deserves further studies. 
a)
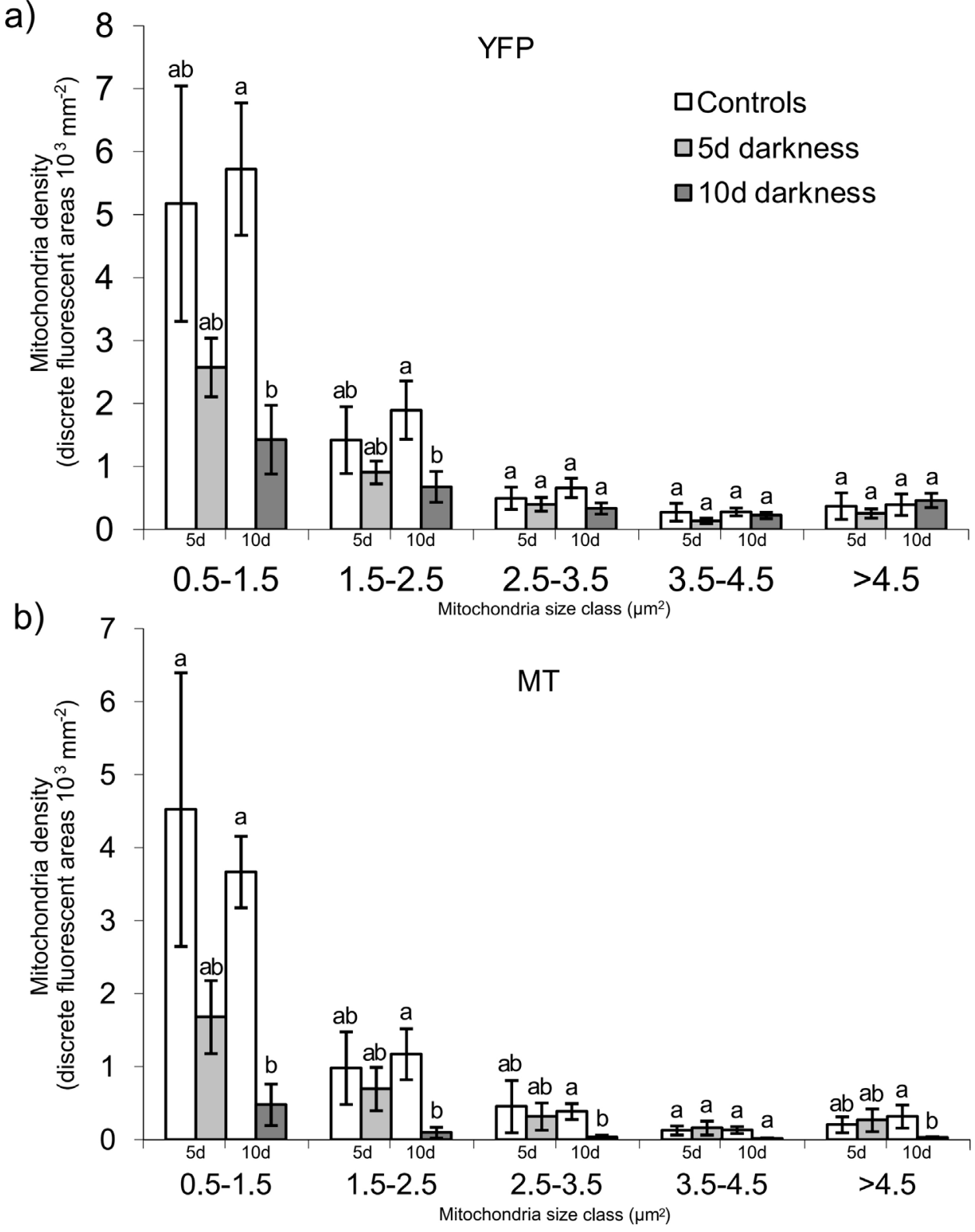

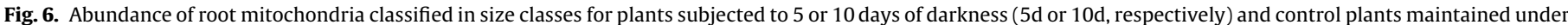

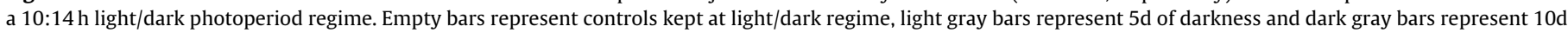
of darkness. a) YFP-labelled mitochondria. b) MT-labelled mitochondria. Different letters indicate significant differences (Kruskal-Wallis test $P \leq 0.05$ ).

\subsection{Size of root mitochondria in dark-incubated plants}

Mitochondria are pleomorphic organelles co-existing as a mixture of heterogeneous morphologies in the same cell [38]. Their different sizes and morphologies seem to be regulated by a particular set of genes [43] which are differentially expressed under different conditions. Three features are evident when mitochondria are observed with fluorescent probes: i) they are not equally distributed in the cytoplasm; ii) they do not show similar size, and iii) they are highly dynamic, changing their forms and positions [44]. The data obtained here demonstrate modifications, especially in root mitochondria size distribution during plant senescence induced by dark treatment to the whole plant.

Mitochondria showed size heterogeneity, with smaller mitochondria being the more abundant and, presumably, the largest contributors to total root mitochondrial activity. Some evidences show that the morphology and size of mitochondria change at the end of the cell cycle $[11,13,20,43]$. These evidences have been obtained using different experimental approaches (e.g., in vitro cell and protoplast cultures, leaf disks, etc.) and with the addition of reactive oxygen species inducers, which may cause discrepancies due to the origin of the plant material (e.g., tissues examined, species, etc.) and mode of culture. However, other results suggest that the size of mitochondria is not affected during the development of leaf epidermal cells [45]. In our experiments, there is a preferential decrease in the amount of the smallest mitochondria, associated with decreased respiration, suggesting that these are the class of mitochondria responsible for most of respiratory activity. On the other hand, it is remarkable that there are not only sets of mitochondria distinguished by their size but, additionally, there are sets of mitochondria possibly with different activity. This is clearly observable by confocal microscopy, with a few or no mitochondria labelled with MT after $10 \mathrm{~d}$ under darkness. It is important to mention that the confocal observations were made on root corti- 

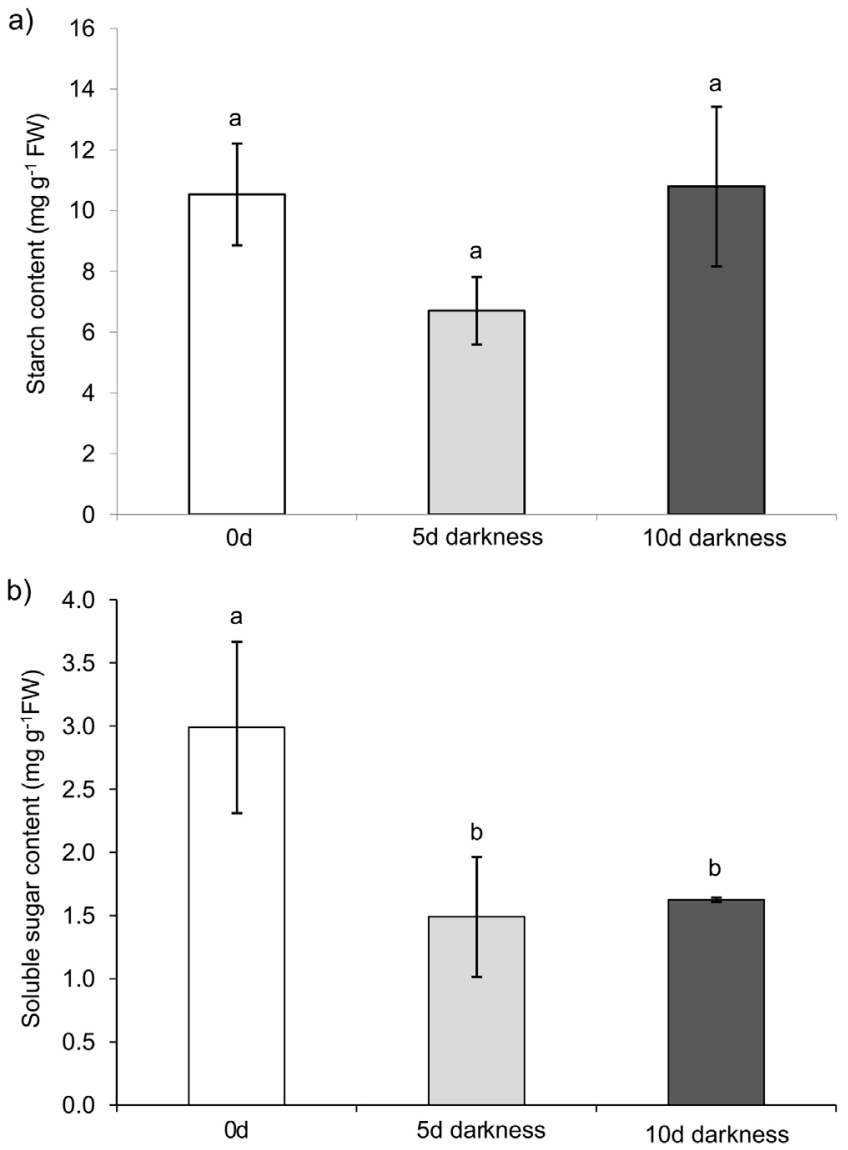

Fig. 7. Contents of starch and soluble sugars in roots of plants subjected to 5 or 10 days of darkness ( $5 \mathrm{~d}$ or $10 \mathrm{~d}$, respectively) and control plants maintained under a 10:14 hs light/dark photoperiod regime. Empty bars represent controls kept at light/dark regime, light gray bars represent $5 \mathrm{~d}$ of darkness and dark gray bars represent $10 \mathrm{~d}$ of darkness. a) Starch content. b) Soluble sugars content. Different letters indicate significant differences (ANOVA $P \leq 0.05$ ). cal sections, and respiratory activity measurements were made on the whole root system. Therefore, other root tissues (e.g., epidermis, phloem) might explain the lack of a full agreement between respiratory activity and the amount of mitochondria with $\Delta \Psi \mathrm{m}$. Moreover, it is interesting to note that differences between total mitochondria amounts (i.e., YFP-labelled) and mitochondria with $\Delta \Psi \mathrm{m}$ point to the presence of a pool of mitochondria with only basal activity or in a "standby mode".

Both mitochondrial number and size depend, in part, on the balance between mitochondrial fission and fusion [38]. These processes are frequent in plant cells [46]. For example, the disruption of either of two Arabidopsis dynamin-like genes, DRP3A or DRP3B, results in an increase in the size of the individual mitochondria and a concomitant decrease in the number of mitochondria per cell $[47,38]$. We found that while smaller mitochondria were the most abundant and variable (i.e., their amount decreased the most after $10 \mathrm{~d}$ under darkness), the abundance of mitochondria of other size classes remained fairly constant. If a fusion process were involved, an increase in the abundance of larger mitochondria should parallel the decrease in the abundance of smaller ones, which does not occur in our experiments. Therefore, it is probable that fusion processes were not involved in roots of darkened plants.

\subsection{The participation of sugars in root responses to whole plant dark treatment}

The early senescence of rosette leaves during post-bolting development in Arabidopsis plants suggests that roots may experience a decrease in activity due to photo-assimilate starvation. The dark treatment of whole plants might reproduce this phenomenon causing a carbohydrate deficit in roots. To test this hypothesis, the contents of starch and soluble sugars in roots of dark treated plants were analyzed. Since starch content didnít show significant differences, but soluble sugars decreased significantly, changes in soluble sugars may be responsible for the drop of mitochondrial activity. Consistently with this, Bigham and Ress [9] obtained similar results with clover roots separated from the plant. These roots maintained their respiratory activity and starch contents for up to $30 \mathrm{~d}$ after detaching the aboveground photosynthetic parts of the plant, while

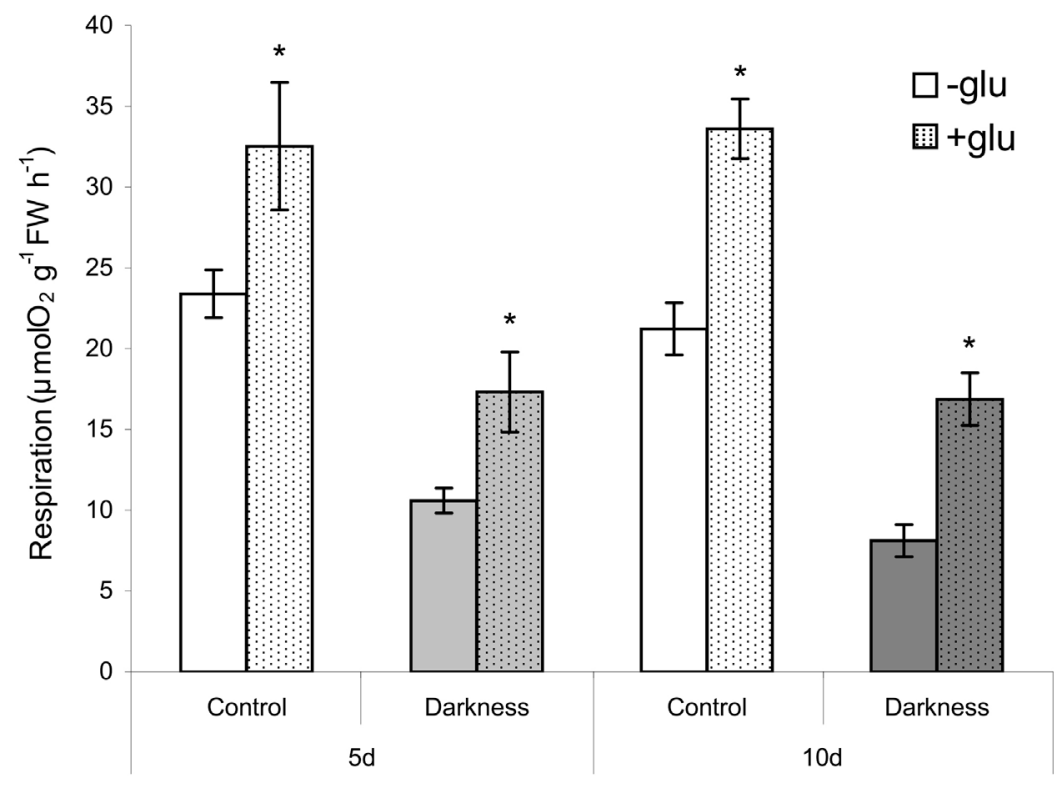

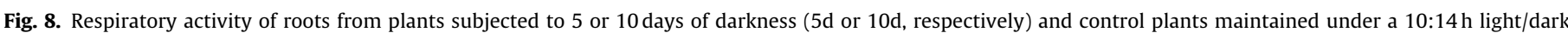

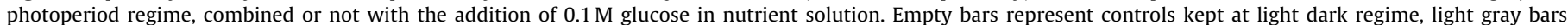

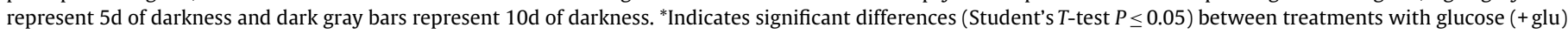
and without glucose (-glu). 
soluble sugar content decreased. A. thaliana mutants lacking functional enzymes related with starch metabolism, either inside the plastid or in the cytosol, show root starch contents similar to wild type and very low compared with leaves [48] suggesting a reduced carbon flux from starch to respiration. In contrast to our results, soybean roots consume all the starch after one day under darkness [49] and sycamore protoplast cultures subjected to sucrose deprivation show a decrease in starch content after $10 \mathrm{~h}$ of starvation [10]. The discrepancies regarding changes in starch content may be the consequence of different experimental approaches and species, while a decrease in soluble sugars is clearly evident in all cases.

The partial reversion of $\mathrm{O}_{2}$ consumption decline in plants incubated in $0.1 \mathrm{M}$ glucose indicates that the deficit of soluble sugars is partly responsible for the decrease in the respiratory activity of roots. Several experimental approaches show increased respiratory activity in carbohydrate starved tissues after carbohydrate addition [10-12,14,50-52]. However, the lack of MT fluorescence in root mitochondria from dark treated roots even after the addition of glucose might be due to the fact that $1 \mathrm{~d}$ incubation may not be enough to restore the $\Delta \Psi \mathrm{m}$. Also, an increase of the specific activity of the pool of mitochondria with $\Delta \Psi \mathrm{m}$ might explain the failure of MT to detect an increase in the abundance of mitochondria with $\Delta \Psi \mathrm{m}$ after glucose supply.

The incubation of roots for $1 \mathrm{~d}$ in $0.1 \mathrm{M}$ sucrose immediately after detachment from the plant prevents the drop in respiration, but the addition of sucrose is ineffective when it is provided $4 \mathrm{~d}$ after organ removal [9]. Similar results were found with sycamore cell cultures [10] and maize roots where respiration could only be restored if glucose $(0.2 \mathrm{M})$ was supplied up to before $4 \mathrm{~d}$ of starvation [12]. Soluble sugars could act in signalling [11,53] of metabolic process aside from acting as a source of energy for metabolic processes. At the concentrations used here $(0.1 \mathrm{M})$, glucose might function primarily as a source of energy, but a signalling function cannot be ruled out.

Because starch apparently is not remobilized, alternative substrates such as proteins or amino acids may be used for respiration. Protein turnover and degradation often occurs concomitantly with chlorophyll and lipid breakdown, at times when plant cells are carbon limited, for example, during extended periods of darkness and leaf senescence, and provide alternative substrates for the mitochondrial electron transport chain [54]. In leaves a functional electron transfer flavoprotein (ETF) is essential for survival of sucrose-depleted cells $[55,56]$. This flavoprotein is involved in the catabolism of Leu and potentially of other amino acids, and it probably supplies mitochondria with respiratory substrates $[55,56]$. Therefore, substrates other than soluble sugars might contribute to root respiration under darkness.

\subsection{Concluding remarks}

The data presented here demonstrate that metabolic activity of roots from naturally senescing plants is maintained beyond the death of aboveground photosynthetic organs. In addition, the decreases in respiration and amount of mitochondrial proteins may be the consequence of a decrease in the abundance of mitochondria, mainly the small ones. The differences between the amount of mitochondria with $\Delta \Psi \mathrm{m}$ and total mitochondria suggest the presence of mitochondria in a "standby state". Part of the drop in root metabolic activity in plants under darkness may be due to the reduced levels of root carbohydrates under such conditions.

\section{Acknowledgements}

DDF is a post-doctoral fellow at CONICET, and CGB and JJG are researchers at CONICET. This work was funded by Universi- dad Nacional de La Plata, Argentina (Grants 11/A258 and 11/A257). The authors are grateful to Dra. María Lorenza Costa (INFIVE) for stimulating discussions of the results and advice on biochemical techniques, and to Dr. Daniel González (Universidad Nacional del Litoral, Santa Fe, Argentina) for providing the anti-COX2 antibody.

\section{Appendix A. Supplementary data}

Supplementary data associated with this article can be found, in the online version, at http://dx.doi.org/10.1016/j.plantsci.2017.01. 013.

\section{References}

[1] L.D. Noodén, The phenomena of senescence and aging, in: L.D. Noodén, A.C. Leopold (Eds.), Senescence and Aging in Plants, Academic Press, San Diego, 1988, pp. 1-50.

[2] M.B. Peoples, M.J. Dalling, The interplay between proteolysis and amino acid metabolism during senescence and nitrogen reallocation, in: L.D. Noodén, A.C. Leoppold (Eds.), Senescence and Aging in Plants, Academic Press, San Diego, 1988, pp. 181-217

[3] C. Masclaux-Daubresse, F. Daniel-Vedele, J. Dechorgnat, F. Chardon, L. Gaufichon, A. Suzuki, Nitrogen uptake, assimilation and remobilization in plants: challenges for sustainable and productive agriculture, Ann. Bot. 105 (2010) 1141-1157

[4] O. Atkin, A. Millar, P. Gardeström, D. Day, Photosynthesis, carbohydrate metabolism and respiration in leaves of higher plants, in: R.C. Leegood, T.D. Sharkey, S. von Caemmerer (Eds.), Photosynth. Physiol. Metab., Kluwer Academic Publishers, Netherlands, 2000, pp. 153-175.

[5] H. Millar, J. Whelan, K.L. Soole, D. Day, Organization and regulation of mitochondrial respiration in plants, Annu. Rev. Plant Biol. 62 (2011) 79-104

[6] J.T. van Dongen, K.J. Gupta, S.J. Ramírez-Aguilar, W.L. Araújo, A. Nunes-Nesi, A.R. Fernie, Regulation of respiration in plants: a role for alternative metabolic pathways, J. Plant Physiol. 168 (2011) 1434-1443.

[7] R.D. Vierstra, Proteolysis in plants: mechanisms and functions, Plant Mol. Biol. 32 (1996) 275-302.

[8] O. Keech, E. Pesquet, A. Ahad, A. Askne, D. Nordvall, S.M. Vodnala, H. Tuominen, V. Hurry, P. Dizengremel, P. Gardeström, The different fates of mitochondria and chloroplasts during dark-induced senescence in Arabidopsis leaves, Plant Cell Environ. 30 (2007) 1523-1534.

[9] I.J. Bingham, R.M. Rees, Senescence and N release from clover roots following permanent excision of the shoot, Plant Soil 303 (2007) 229-240.

[10] E.P. Journet, R. Bligny, R. Douce, Biochemical changes during sucrose deprivation in higher plant cells, J. Biol. Chem. 261 (1986) 3193-3199.

[11] P. Giege, Coordination of nuclear and mitochondrial genome expression during mitochondrial biogenesis in Arabidopsis, Plant Cell 17 (2005) 1497-1512.

[12] R. Brouquisse, F. James, P. Raymond, A. Pradet, Study of glucose starvation in excised maize root tips, Plant Physiol. 96 (1991) 619-626.

[13] M. Zottini, E. Barizza, F. Bastianelli, F. Carimi, F. Lo Schiavo, Growth and senescence of Medicago truncatula cultured cells are associated with characteristic mitochondrial morphology, New Phytol. 172 (2006) 239-247.

[14] I. Couée, M. Jan, J.P. Carde, R. Brouquisse, P. Raymond, A. Pradet, Effects of glucose starvation on mitochondrial subpopulations in the meristematic and submeristematic regions of maize root, Plant Physiol. 100 (1992) 1891-1900.

[15] D.C. Logan, Having a swell time - mitochondrial morphology and plant cell death programmes, J. Microsc. 231 (2008) 215-224

[16] J.M. Mach, A.R. Castillo, R. Hoogstraten, J.T. Greenberg, The Arabidopsis-accelerated cell death gene ACD2 encodes red chlorophyll catabolite reductase and suppresses the spread of disease symptoms, Proc. Natl. Acad. Sci. U. S. A. 98 (2001) 771-776.

[17] M.J. Curtis, T.J. Wolpert, The oat mitochondrial permeability transition and its implication in victorin binding and induced cell death, Plant J. 29 (2002) 295-312.

[18] K. Yoshinaga, S.I. Arimura, Y. Niwa, N. Tsutsumi, H. Uchimiya, M. Kawai-Yamada, Mitochondrial behaviour in the early stages of ROS stress leading to cell death in Arabidopsis thaliana, Ann. Bot. 96 (2005) 337-342.

[19] K. Yoshinaga, M. Fujimoto, S.I. Arimura, N. Tsutsumi, H. Uchimiya, M. Kawai-Yamada, The mitochondrial fission regulator DRP3B does not regulate cell death in plants, Ann. Bot. 97 (2006) 1145-1149.

[20] I. Scott, D.C. Logan, Mitochondrial morphology transition is an early indicator of subsequent cell death in Arabidopsis, New Phytol. 177 (2008) 90-101.

[21] S. Arpagaus, A. Rawyler, R. Braendle, Occurrence and characteristics of the mitochondrial permeability transition in plants, J. Biol. Chem. 277 (2002) 1780-1787.

[22] L.D. Noodén, J.J. Guiamét, I. John, Senescence mechanisms, Physiol. Plant. 101 (1997) 746-753.

[23] B.K. Nelson, X. Cai, A. Nebenführ, A multicolored set of in vivo organelle markers for co-localization studies in Arabidopsis and other plants, Plant J. 51 (2007) 1126-1136. 
[24] H. Norén, P. Svensson, B. Andersson, A convenient and versatile hydroponic cultivation system for Arabidopsis thaliana, Physiol. Plant. 121 (2004) $343-348$.

[25] J.E. Leggett, M.H. Frere, Growth and nutrient uptake by soybean plants in nutrient solutions of graded concentrations, Plant Physiol. 48 (1971) 457-460.

[26] C.G. Bartoli, M. Simontacchi, J.J. Guiamet, E. Montaldi, S. Puntarulo, Antioxidant enzymes and lipid peroxidation during aging of Chrysanthemum morifolium RAM petals, Plant Sci. 104 (1995) 161-168.

[27] U.K. Laemmli, Cleavage of structural proteins during assembly of head of bacteriophage T4, Nature 227 (1970) 680-685.

[28] C.V. Attallah, E. Welchen, A.P. Martin, S.V. Spinelli, G. Bonnard, J.F. Palatnik, D.H. Gonzalez, Plants contain two SCO proteins that are differentially involved in cytochrome $c$ oxidase function and copper and redox homeostasis, J. Exp. Bot. 62 (2011) 4281-4294.

[29] C.G. Bartoli, J.J. Guiamet, G. Kiddle, G.M. Pastori, R. Di Cagno, F.L. Theodoulou, C.H. Foyer, Ascorbate content of wheat leaves is not determined by maximal L-galactono-1,4-lactone dehydrogenase (GalLDH) activity under drought stress, Plant Cell Environ. 28 (2005) 1073-1081.

[30] A. Macho, D. Decaudin, M. Castedo, T. Hirsch, S. a. Susin, N. Zamzami, G. Kroemer, Chloromethyl-X-rosamine is an aldehyde-fixable potential-sensitive fluorochrome for the detection of early apoptosis, Cytometry 25 (1996) 333-340.

[31] K. Wakamatsu, M. Fujimoto, M. Nakazono, S.I. Arimura, N. Tsutsumi, Fusion of mitochondria in tobacco suspension cultured cells is dependent on the cellular ATP level but not on actin polymerization, Plant Cell Rep. 29 (2010) 1139-1145.

[32] S. Bolte, F.P. Cordelières, A guided tour into subcellular colocalisation analysis in light microscopy, J. Microsc. 224 (2006) 13-232.

[33] E.W. Yemm, A.J. Willis, The estimation of carbohydrates in plant extracts by anthrone, Biochem. J. 57 (1954) 508-514.

[34] M.L. Costa, P.M. Civello, A.R. Chaves, G.A. Martínez, Effect of hot air treatments on senescence and quality parameters of harvested broccoli (Brassica oleraced L var Italica) heads, J. Sci. Food Agric. 85 (2005) 1154-1160.

[35] R. Rose, C.L. Rose, S.K. Omi, K.R. Forry, D.M. Durall, W.L. Bigg, Starch determination by perchloric acid vs enzymes: evaluating the accuracy and precision of six colorimetric methods, J. Agric. Food Chem. 39 (1991) 2-11.

[36] D. Chrobok, S.R. Law, B. Brouwer, P. Lindén, A. Ziolkowska, D. Liebsch, R. Narsai, B. Szal, T. Moritz, N. Rouhier, J. Whelan, P. Gardeström, O. Keech, Dissecting the metabolic role of mitochondria during developmental leaf senescence, Plant Physiol. 172 (2016) 2132-2153.

[37] C.P. Lee, G. Maksaev, G.S. Jensen, M.W. Murcha, M.E. Wilson, M. Fricker, R. Hell, E.S. Haswell, A.H. Millar, L.J. Sweetlove, MSL1 is a mechanosensitive ion channel that dissipates mitochondrial membrane potential and maintains redox homeostasis in mitochondria during abiotic stress, Plant J. 88 (2016) $809-825$.

[38] I. Scott, D.C. Logan, Mitochondrial dynamics: the control of mitochondrial shape, size, number, motility, and cellular inheritance, in: D.C. Logan (Ed.), Plant Mitochondria, Blackwell Publishing Ltd., 2007, pp. 1-36.

[39] K.L. Griffin, O.R. Anderson, M.D. Gastrich, J.D. Lewis, G. Lin, W. Schuster, J.R. Seemann, D.T. Tissue, M.H. Turnbull, D. Whitehead, Plant growth in elevated $\mathrm{CO}_{2}$ alters mitochondrial number and chloroplast fine structure, Proc. Natl. Acad. Sci. U. S. A. 98 (2001) 2473-2478.
[40] D.A. Day, A.H. Millar, J.T. Wiskich, J. Whelan, Regulation of alternative oxidase activity by pyruvate in soybean mitochondria, Plant Physiol. 106 (1994) 1421-1427.

[41] C. Hiser, L. McIntosh, Alternative oxidase of potato is an integral membrane protein synthesized de novo during aging of tuber slices, Plant Physiol. 93 (1990) 312-318.

[42] G.C. Vanlerberghe, C.A. Robson, J.Y.H. Yip, Induction of mitochondrial alternative oxidase in response to a cell signal pathway down-regulating the cytochrome pathway prevents programmed cell death, Plant Physiol. 129 (2002) 1829-1842

[43] D.C. Logan, Mitochondrial dynamics, New Phytol. 160 (2003) 463-478.

[44] D.C. Logan, C.J. Leaver, Mitochondria-targeted GFP highlights the heterogeneity of mitochondrial shape, size and movement within living plant cells, J. Exp. Bot. 51 (2000) 865-871.

[45] A.F. Armstrong, D.C. Logan, O.K. Atkin, On the developmental dependence of leaf respiration: responses to short- and long-term changes in growth temperature, Am. J. Bot 93 (2006) 1633-1639.

[46] S. Arimura, J. Yamamoto, G.P. Aida, M. Nakazono, N. Tsutsumi, Frequent fusion and fission of plant mitochondria with unequal nucleoid distribution, Proc. Natl. Acad. Sci. U. S. A. 101 (2004) 7805-7808.

[47] S. Arimura, N. Tsutsumi, A dynamin-like protein (ADL2b) rather than FtsZ, is involved in Arabidopsis mitochondrial division, Proc. Natl. Acad. Sci. U. S. A. 99 (2002) 5727-5731.

[48] I. Malinova, M. Steup, J. Fettke, Starch-related cytosolic heteroglycans in roots from Arabidopsis thaliana, J. Plant Physiol. 168 (2011) 1406-1414.

[49] P.S. Kerr, T.W. Rufty, S.C. Huber, Changes in nonstructural carbohydrates in different parts of soybean (Glycine max [L.] Merr.) plants during a light/dark cycle and in extended darkness, Plant Physiol. 78 (1985) 576-581.

[50] C. Roby, J.-B. Martin, R. Bligny, R. Douce, Biochemical changes during sucrose deprivation in higher plant cells, J. Biol. Chem. 262 (1987) 5000-5007.

[51] R. Brouquisse, J.-P. Gaudillère, P. Raymond, Induction of a carbon-starvation-related proteolysis in whole maize plants submitted to light/dark cycles and to extended darkness, Plant Physiol. 117 (1998) 1281-1291.

[52] R. Brouquisse, D. Rolin, S. Cortès, M. Gaudillère, A. Evrard, C. Roby, A metabolic study of the regulation of proteolysis by sugars in maize root tips: effects of glycerol and dihydroxyacetone, Planta 225 (2007) 693-709.

[53] F. Rolland, E. Baena-Gonzalez, J. Sheen, Sugar sensing and signaling in plants: conserved and novel mechanisms, Annu. Rev. Plant Biol. 57 (2006) 675-709.

[54] W.L. Araújo, T. Tohge, K. Ishizaki, C.J. Leaver, A.R. Fernie, Protein degradation an alternative respiratory substrate for stressed plants, Trends Plant Sci. 16 (2011) 489-498.

[55] K. Ishizaki, T.R. Larson, N. Schauer, A.R. Fernie, I.A. Graham, The critical role of Arabidopsis electron-transfer flavoprotein: ubiquinone oxidoreductase during dark-induced starvation, Plant Cell 17 (2005) 2587-2600.

[56] K. Ishizaki, N. Schauer, T.R. Larson, I.A. Graham, A.R. Fernie, C.J. Leaver, The mitochondrial electron transfer flavoprotein complex is essential for survival of Arabidopsis in extended darkness, Plant J. 47 (2006) 751-760. 